P79 (continued)

analyses indicated that the association was stronger between VAI and T2D in Qatari women than in men.

Conclusions: VAI was a strong and independent predictor of T2D among the Qatari adult population. Therefore, VAI could be a useful tool for predicting the risk of T2D among Qatari adults.

Funding: Qatar University.

\section{P80 The Effect of Attendance on Anthropometric Outcomes of Children Completing a Pediatric Weight Management Program for Latino Families}

Evelyn Zavala, BS, George Mason University, Global \& Community Health; Daisy Posada, MPH, BS, George Mason University, Global \& Community Health; Margaret $T$. Jones, PhD, George Mason University, Kinesiology; Robyn Mehlenbeck, PhD, ABPP, George Mason University, Psychology; Sina Gallo, PhD, RDN, MS, sgallo@gmu.edu, George Mason University, Nutrition \& Food Studies, 4408 Patriot Circle Suite 4100 MSN 1F7, Fairfax, VA, 22030

Background: Latinos have some of the highest rates of pediatric obesity compared to other ethnic minorities. Treatment efforts should include a multidisciplinary and culturally adapted approach. Program attendance has been shown to influence health outcomes, yet has not been explored with pediatric programs targeting lowincome and ethnic minority populations.

Objective: To evaluate whether attendance improves anthropometric outcomes among Latino children who are overweight/obese and participating in a family based pediatric weight management program.

Study Design, Setting, Participants: Latino children participated in Vidas Activas y Familias Saludables (VALÉ), a multidisciplinary group-based program which focuses on diet, exercise, behavior modification, and adaptation to the Latino community. Latino children aged 5 to 9 years with BMI-for-age $\geq 85$ th percentile (overweight/obese category) were recruited through clinics and schools between 2017 and 2019. Participants and their families met approximately 90 minutes per week over the 10-week intervention.

Measurable Outcome/Analysis: Family attendance at each session was recorded. Anthropometric measurements including waist circumference, body fat (using bioelectrical impedance), height and weight for the assessment of BMI-for-age Z scores (compared to CDC growth charts) were measured pre-, and immediately-post the intervention. As data were non-normally distributed the relationship between attendance and anthropometric outcomes were analyzed via SPSS using Spearman correlation and Kruskal Wallis $\mathrm{H}$ test.

Results: A total of 57 participants completed both pre and post assessments with 7 who attended $0-40 \%$ of sessions, 12 attended 50-60\%, 16 attended $70-80 \%$, and 22 attended $\geq 90 \%$. Although the median change in BMI, BMI Z, and waist circumference improved with higher attendance, no significant associations were observed between number of sessions attended and anthropometric outcomes.

Conclusions: Higher program attendance did not affect anthropometric outcomes of children participating in pediatric weight management program targeting lowincome Latino families. The limited sample size available may have affected these results.

Funding: George Mason University; Potomac Health Foundation.

\section{P81 Development and Reliability Testing of a Nutrition Knowledge Questionnaire for Australian Children (CNK-AU)}

Nienke de Vlieger, MS, nienke.devlieger@uon.edu.au, University of Newcastle, University Dr, Callaghan, NSW, Australia, 2308; Nick Riley, PhD, University of Newcastle; Andrew Miller, PhD, University of Newcastle; Clare Collins, PhD, University of Newcastle; Tamara Bucher, PhD, University of Newcastle

Background: Increased nutrition knowledge is related to better eating behavior. However, internationally, there are few reliable and validated tools to measure the nutrition knowledge of children, and a tool aimed at Australian children is lacking entirely.

Objective: Developing a valid and reliable tool that can be used by practitioners and researchers to assess children's level of nutrition knowledge.

Study Design, Setting, Participants: A team of nutrition and education experts developed a nutrition knowledge survey for Australian children aged 8-12 years, based on an existing and validated Belgian survey. The questions were translated and adapted to confirm with Australian culture and governmental recommendations. It consists of 8 nutrition-related categories: 'healthy choices', 'portion and serve sizes', 'balanced meals', 'nutrition labels', 'nutrient functions' and food- 'sources', 'safety' and 'categories'. Year 5 and 6 students $(\mathrm{n}=94$, age $=10.9$, $\mathrm{SD}=0.76)$ at a primary school in Newcastle, Australia were recruited and completed the nutrition knowledge survey twice, with one week in between. The questionnaire was filled out online, using an Android tablet. Teachers were asked not to teach any nutrition between the measurements.

Measurable Outcome/Analysis: Item analysis showed varied item difficulty and discrimination. The interrater reliability per item between $\mathrm{T} 0$ and $\mathrm{T} 1$, was moderate to substantial for the majority of the items (mean $\kappa=0.50$, $\mathrm{SD}=0.21)$. Test retests showed a significant correlation for the total score $(\mathrm{r}=.756)$ and all categories except 'Portion and serve sizes' ('healthy choices' $[\mathrm{r}=.616]$, 'balanced meals' $[\mathrm{r}=.585]$, 'nutrition labels' $[\mathrm{r}=.595]$, 'nutrient functions' $[\mathrm{r}=.582]$ and food- 'sources' $[\mathrm{r}=.455]$, 'safety' $[\mathrm{r}=.502]$ and 'categories' $[\mathrm{r}=.825])$.

Conclusions: The CNK-AU was found to be a practical and reliable tool for measuring Australian children's nutrition knowledge. The tool may be useful in future work to determine the effectiveness of educational interventions and can be adapted to other cultures.

Funding: None. 